\title{
Condições de saúde e utilização de um serviço de atenção primária em pacientes hipertensos e/ou diabéticos
}

\author{
Health conditions and utilization of primary health care service in patients \\ with hypertension and diabetes
}

\author{
Sara Bortoluza, Lena Azeredo de Limab ${ }^{\mathrm{b}}$ Fúlvio Borges Nedelc \\ a Nutricionista. Especialização em Saúde da Família e Comunidade pelo Grupo Hospitalar Conceição e Especialista em Nutrição Clínica Personalizada. \\ b Nutricionista. Mestre em Epidemiologia, Faculdade de Medicina, Universidade Federal do Rio Grande do Sul Brasil. Centro de Estudo e Pesquisa em \\ Atenção Primária à Saúde (CEPAPS/GHC); Serviço de Saúde Comunitária do Grupo hospitalar Conceição (SSC/GHC). \\ Médico. Doutor em Epidemiologia, Universidade Federal de Pelotas (UFPel). Departamento de Saúde Pública, Centro de Ciências da Saúde, Universidade Federal \\ de Santa Catarina - SPB/CCS/UFSC; Grups de Recerca d'Amèrica i Âfrica Llatines, Unitat de Bioestadística, Universitat Autònoma de Barcelona - GRAAL/UAB.
}

RESUMO

Objetivos: Caracterizar condições sociodemográficas e de saúde, hábitos, uso e percepção da qualidade dos serviços de saúde dos hipertensos e/ou diabéticos usuários do Serviço de Saúde Comunitária do Grupo Hospitalar Conceição.

Materiais e Métodos: Análise univariada transversal do perfil sociodemográfico, fatores de risco para doenças cardiovasculares, percepção de saúde, utilização e satisfação com o serviço de saúde de uma amostra aleatória de pessoas com hipertensão e/ou diabetes acompanhados pelo Serviço de Saúde Comunitária do Grupo Hospitalar Conceição, que responderam a um questionário estruturado com perguntas fechadas, aplicado em entrevista domiciliar por entrevistadores treinados.

Resultados: Dos 2.482 analisados, 67\% eram apenas hipertensos, 6\% apenas diabéticos e 27\% apresentavam as duas condições. Eram em maioria mulheres (68\%), idosos (60\%), com escolaridade até o ensino fundamental completo (78\%) e grande parte considerou sua saúde regular (42\%) ou boa (41\%), sendo que $11 \%$ consideraram ruim. Quanto aos fatores de risco, 15\% eram fumantes, 30\% apresentavam alimentação inadequada, metade eram sedentários e $70 \%$ apresentavam excesso de peso, sendo $31 \%$ obesos. Mais de $63 \%$ consultou com o médico pelo menos uma vez nos últimos seis meses, mas o acompanhamento regular por outros profissionais ou a participação em grupos é baixa. Conclusão: Conclui-se que o cuidado com os fatores de risco relacionados com estilo de vida pode ser intensificado, através da melhor utilização dos recursos da unidade de saúde, como participação em grupos, visitas domiciliares e consultas com os enfermeiros e dentistas.

Palavras-chave: doença crônica; acesso aos serviços de saúde; satisfação do paciente; atenção primária à saúde.

Objectives: To characterize socio-demographic and health conditions, habits, use and perception of quality of health services of hypertensive and diabetic users of Community Health Service (Conceição Hospital Group).

Materials and Methods: Cross univariate analysis of the socio-demographic profile, risk factors for cardiovascular disease, health perception, use and satisfaction with the health service of a random sample of people with hypertension or diabetes accompanied by Community Health Service (Conceição Hospital Group), who responded to a structured questionnaire with closed questions, applied in home interviews by trained interviewers.

Results: Of the 2,482 analyzed $67 \%$ were only hypertensive, $6 \%$ only diabetics and $27 \%$ had both conditions. They were mostly women (68\%), elderly (60\%), with education until complete elementary education (78\%) and most of them considered their health as regular (42\%) or good (41\%) and 11\% considered their health as poor. As for risk factors, $15 \%$ were smokers, $30 \%$ had inadequate nutrition, half were sedentary, $70 \%$ were overweight and 31\% obese. More than $63 \%$ consulted with a doctor at least once in the last six months, but regular monitoring by other professionals or group membership is low.

Conclusion: We conclude that the care with the risk factors related to lifestyle can be enhanced through the better use of resources of the health unit, such as group membership, home visits and consultations with nurses and dentists.

Keywords: chronic disease; access to health services; patient satisfaction; primary health care. 


\section{INTRODUÇÃO}

As doenças crônicas não transmissíveis (DCNT) são as principais causas de morte no mundo, tanto em países desenvolvidos quanto naqueles em desenvolvimento ${ }^{1}$. Aproximadamente, $80 \%$ dos óbitos por DCNT ocorrem em países de renda baixa ou média, onde $29 \%$ desses registros são de pessoas com menos de 60 anos, enquanto nos países de alta renda, apenas 13\% são óbitos precoces². Em 2008, as DCNT foram responsáveis por $63 \%$ dos óbitos no mundo e a projeção para o ano de 2020 é que estejam relacionadas a $73 \%$ das mortes ${ }^{3,4}$.

O número de diabéticos está aumentando em virtude do crescimento e do envelhecimento populacional, da maior urbanização, da crescente prevalência de obesidade e sedentarismo, bem como a maior sobrevida de pacientes com Diabetes Mellitus (DM). A prevalência na população adulta no Brasil é de $7,6 \% 5$, chegando a $13,5 \%$ para o estado de São Paulo ${ }^{6}$. Observou-se incremento de $2,7 \%$ na faixa etária de 30 a 59 anos para $17,4 \%$ na de 60 a 69 anos $^{6}$. Em comparação com outros países, há grandes diferenças na prevalência, praticamente metade da população adulta apresenta DM em Nauru, na Oceania e entre os índios Pima, no Arizona, nos Estados Unidos ${ }^{6}$. A Organização Mundial da Saúde (OMS) estima que o número total de pessoas com DM no mundo elevar-se-á, de 171 milhões, em 2000, para 366 milhões, em 2030, e no Brasil, de 4,5 milhões para 11,3 milhões, no mesmo período. A prevalência da doença deve crescer, entre 1995 e 2025, em 48\% nos países em desenvolvimento, contra $27 \%$ nos países desenvolvidos ${ }^{7}$. Ademais, a hipertensão afeta $40 \%$ ou mais dos indivíduos diabéticos ${ }^{2}$.

A prevalência de Hipertensão (HAS) varia de $22 \%$ a $44 \%$ para adultos e tende a aumentar com a idade, chegando aos $50 \%$ entre 60 e 69 anos e $75 \%$ acima de 70 anos. Apresenta baixo índice de controle (19,3\%), sendo responsável por altas taxas de mortalidade por doenças do sistema circulatório ${ }^{8}$. Ao analisar as 17 capitais brasileiras e o Distrito Federal, a frequência de HAS auto-referida variou de $18 \%$ a $29 \%{ }^{5}$. Entre as diferentes regiões do país, a prevalência na Região Nordeste é de 5,04 a 37,9\%, na Região Sudeste é de 5,04 a $37,9 \%$, na Região Sul é de 1,28 a $27,1 \%$ e na Região Centro-Oeste é de 6,3 a 16,75\% .

O controle dessas condições nos indivíduos afetados isto é, a manutenção dos níveis tensionais ou glicêmicos em determinados limites de normalidade - depende de uma série de fatores, desde a adoção de estilos de vida que envolva atividade física e dieta adequada ao uso continuado de medicamentos, sendo assim fortemente determinado pelas condições de vida e acesso a serviços de saúde de qualidade $3,10,11$. Dada a cronicidade dessas condições e a complexidade de seu controle, a Atenção Primária à Saúde (APS) é a principal instância de atenção a esses pacientes ${ }^{12,13}$.

Com a Estratégia Saúde da Família (ESF), iniciada em 1994, o Brasil experimentou um grande avanço na atenção primária à saúde (APS), com a presença de Equipes de Saúde da Família (EqSF) em quase todos os municípios do país, embora a cobertura populacional da ESF esteja em torno de 60\% (DAB/SAS/MS). À diferença da Atenção Básica "Tradicional", a ESF se organiza por equipes multiprofissionais generalistas com responsabilidade sobre uma população definida territorialmente, a qual deve acompanhar longitudinalmente. Entre as inspirações para o ideário da ESF está a experiência do Serviço de Saúde Comunitária do Grupo Hospitalar Conceição (SSC/GHC) em Porto Alegre que, desde a década de 1980, trabalha em APS com equipes multiprofissionais de prática generalista longitudinal sobre uma população definida.

O SSC/GHC conta com 12 unidades de saúde e 40 equipes de saúde da família, localizados em territórios delimitados da Zona Norte de Porto Alegre, servindo de referência para uma população de aproximadamente 108.560 pessoas (IBGE 2010). É composto por equipes multiprofissionais incluindo assistentes sociais, enfermeiros, farmacêuticos, médicos de família e comunidade, nutricionistas, psicólogos e agentes de saúde. Conta com um programa de Residência em Medicina de Família e Comunidade há mais de 35 anos e um de Residência Integrada em Saúde (multiprofissional com ênfase em Saúde da Família e Comunidade), implantado em 2004.

Assim, estudos sobre o SSC/GHC ou a população sob sua responsabilidade podem contribuir para a solução de dificuldades encontradas na ESF. Entre as dificuldades mais relatadas em relação ao acesso em Unidades de Saúde no Brasil, encontram-se a obtenção de consulta de clínica médica e odontológica, além do tempo de espera para agendamento e a consulta ${ }^{14}$. Sendo assim, o objetivo desta pesquisa foi caracterizar condições sociodemográficas e de saúde, hábitos, uso e percepção da qualidade dos serviços de saúde dos hipertensos e/ou diabéticos usuários do Serviço de Saúde Comunitária do Grupo Hospitalar Conceição.

\section{MATERIAIS E MÉTODOS}

Trata-se de estudo transversal de uma amostra aleatória de usuários com hipertensão e/ou diabetes acompanhados pelo Serviço de Saúde Comunitária do Grupo Hospitalar Conceição. Foi selecionada uma amostra aleatória simples de 3.784 sujeitos entre os usuários adultos registrados no HiperDia e que estiveram em consulta médica no serviço 
nos últimos três anos. Entre os selecionados, 1.286 usuários foram excluídos do estudo pelos seguintes motivos: recusas; não serem hipertensos nem diabéticos, não residir mais na área de abrangência, ou por ter falecido; estarem de férias ou hospitalizadas; por não localizar alguém em casa após quatro tentativas de visitas no endereço ou ninguém, entre os vizinhos, as conheciam, ou ainda, por não existir o endereço. Após perdas, recusas e exclusões por não pertencer mais à população alvo ou declarar não ter HAS ou DM, resultou em 2.482 sujeitos, numa taxa de resposta estimada em cerca de $74 \%$.

Esta pesquisa trata-se de uma análise de uma seleção de variáveis coletadas na linha de base de um estudo maior intitulado "Avaliação da Atenção à Saúde em Hipertensão e Diabetes na Atenção Primária", desenvolvido pelo Centro de estudo e pesquisa em atenção primária à saúde (Cepaps) do SSC-GHC, o qual teve início em 2011, após ter sido aprovado pelo Comitê de Ética em Pesquisa do GHC, mediante registro 10-261. Foram coletados dados do perfil sociodemográfico, condições de saúde, fatores de risco para doenças cardiovasculares e utilização dos serviços de saúde por meio de um questionário estruturado com perguntas fechadas, aplicado em entrevista domiciliar por entrevistadores treinados. Foi realizada análise descritiva dos dados através da frequência com intervalo de confiança de uma ampla gama de variáveis dividida em 4 blocos:

Condições Sociodemográficas: Foram coletadas informações quanto ao: sexo; idade (calculada em anos completos a partir da data de nascimento e categorizada nas faixas etárias "20-39 anos", "40-59 anos", "60-74 anos", "75-84 anos" e "85-101 anos"); escolaridade, categorizada em "até fundamental completo/médio incompleto" e "médio completo/superior", a partir da resposta de "até que série ou ano completou na escola". Para a classificação socioeconômica utilizou-se o critério da Associação Brasileira de Empresas de Pesquisa (ABEP), agrupado posteriormente em três categorias $(A B, C, D E)$. Houve uma falha no questionário e não foi coletada a escolaridade do chefe da família, item do algoritmo proposto. Para contornar o problema, foi realizada uma coleta por telefone, em que a taxa de resposta foi de 50\%. Estimou-se a concordância entre a classificação ABEP calculada com a escolaridade do chefe da família e aquela calculada com a escolaridade do entrevistado, que para a classificação ABEP agrupada em 3 categorias encontrou índice Kappa 0,862, (IC95 0,8340,893). A situação conjugal foi perguntada e posteriormente classificada em "com companheiro" e "sem companheiro". A convivência na mesma residência com outra pessoa com HAS ou DM foi feita através de pergunta direta e utilizada como dicotômica sim e não.
Condições de Saúde: presença de HAS e presença de DM foram obtidas a partir de duas perguntas realizadas no início do questionário: "Se a pessoa usava remédio para a pressão alta ou alguma vez o médico lhe disse que tinha pressão alta" (idem para DM). Quem respondia "não" para as duas perguntas parava de responder ao questionário e quem respondia "sim" para pelo menos uma das duas, continuava. O problema de saúde foi categorizado em "HAS", "DM" e "HAS*DM". O tempo de diagnóstico do problema foi questionado diretamente em anos e categorizado em "até 1 ano", "até 2 anos" e "acima de 2 anos". A percepção de saúde foi autorreferida pelo entrevistado entre as categorias "excelente", "muito boa", "boa", "regular" e "ruim". A ocorrência de complicações (infarto do coração, derrame cerebral e/ou amputação de algum membro) foi questionada diretamente e utilizada como dicotômica sim e não.

Fatores de risco para Doenças Cardiovasculares: Foram coletados dados sobre: tabagismo, sendo categorizado em "não, nunca fumei", "já fumei, mas parei de fumar" e "sim, fumo (mais de 1 cigarro por dia há mais de 1 mês)"; a adequação alimentar, por meio do teste "Como está a sua alimentação?" do Guia Alimentar de bolso ${ }^{15}$, sendo classificada em três níveis ("adequada", "parcialmente adequada" e "inadequada"); uso problemático de bebida alcoólica, classificado de acordo com o questionário Cut-down; Annoyed; Guilty \& Eye-opener (CAGE) ${ }^{16}$ e classificado em "sim" e "não"; sedentarismo, avaliado de acordo com o questionário International Physical Activity Questionnaires (IPAQ) ${ }^{17}$ e classificado em "sim" e "não"; estado nutricional, avaliado por meio do cálculo do Índice de Massa Corporal (IMC) e classificado conforme OMS ${ }^{18}$, foi categorizado em baixo peso $\left(14,1-18,4 \mathrm{~kg} / \mathrm{m}^{2}\right)$, eutrofia $\left(18,5-24,9 \mathrm{~kg} / \mathrm{m}^{2}\right)$, pré-obeso $\left(25-29,9 \mathrm{~kg} / \mathrm{m}^{2}\right)$, obesidade I (30-34,9 kg/m²) e obesidade II $\left(\geq 35 \mathrm{~kg} / \mathrm{m}^{2}\right)$.

Utilização dos serviços de saúde: Foram coletados dados sobre: o número de consultas médicas e o de consultas com o dentista nos últimos seis meses foram obtidos através de pergunta direta e categorizados em "nenhuma", "1 consulta", "2 consultas", "3-6 consultas" e " $\geq 7$ consultas" com o médico e ter consultado ou não com o dentista; o tempo transcorrido entre o agendamento e a consulta médica foi avaliado através do número de dias relatado pelo entrevistado e categorizado em "até 15 dias" e "mais de 15 dias"; o acompanhamento regular com enfermeiro e nutricionista, ter recebido visita domiciliar de um profissional de saúde no último mês e a participação em grupos de hipertensão ou diabete foram obtidos através de perguntas diretas e utilizadas como dicotômica sim e não. A satisfação com o 
atendimento na unidade de saúde foi avaliada através de uma escala de pontuação de 0-10 e categorizada em "ruim (nota 0-5)", "regular (nota 6-7)" e "bom (nota 8-10)".

A análise dos dados foi feita utilizando os softwares SPSS $^{\circledR}$ versão 18 (Statistical Package for the Social Sciences) e $R$ versão 3.2.3. Os intervalos de confiança foram calculados através da aproximação à binominal pelo método de Moivre ou por métodos exatos, quando a proporção da categoria era menor que 0,2 ou maior que 0,8 .

O projeto de pesquisa foi submetido à apreciação no Comitê de Ética em Pesquisa do Hospital Nossa Senhora da Conceição (HNSC/GHC) e aprovado em 05 de fevereiro de 2014 sob o parecer no 522.048 .

\section{RESULTADOS}

A Tabela 1 apresenta os resultados do bloco das condições socioeconômicas, onde se verificou que a maioria dos usuários eram mulheres e idosos. Em relação à escolaridade, $78 \%$ dos participantes estudaram até o ensino fundamental completo. Quanto à classificação socioeconômica, segundo o critério ABEP modificado, a maioria dos entrevistados pertencia à Classe C. Pouco mais da metade vivia com um companheiro.

A caracterização do problema de saúde dos indivíduos está demonstrada na Tabela 2. Observou-se que $67 \%$ dos usuários tinham hipertensão sem diabetes, $6 \%$ eram somente diabéticos e $27 \%$ apresentavam as duas condições associadas. O tempo de diagnóstico foi superior a dois anos para a maior parte dos hipertensos e diabéticos. A convivência na mesma residência com pessoas do mesmo diagnóstico foi de $46 \%$ para os hipertensos e $24 \%$ para os diabéticos. Houve complicações (infarto agudo do miocárdio, acidente vascular cerebral e amputação em diabéticos) em 16\% dos indivíduos. Grande parte considerou sua saúde regular (42\%) ou boa $(41 \%)$, sendo que $11 \%$ consideraram ruim.

A Tabela 3 mostra os fatores de risco para doenças cardiovasculares relacionados ao estilo de vida dos hipertensos e diabéticos, $15 \%$ eram fumantes, $23 \%$ apresentaram alimentação adequada, 46\% parcialmente adequada e $30 \%$ inadequada, $5 \%$ faziam uso problemático do álcool e 52\% eram sedentários. Em relação ao estado nutricional, 29\% estavam eutróficos, 39\% com sobrepeso e $30 \%$ com obesidade.

Tabela 1. Descrição socioeconômica e demográfica dos hipertensos e diabéticos atendidos no SSC/GHC.

\begin{tabular}{|c|c|c|c|}
\hline Características & Frequência (n) & Percentual (\%) & $\begin{array}{l}\text { Intervalo de Confiança } \\
\qquad(95 \%)\end{array}$ \\
\hline Sexo & 2482 & & \\
\hline Feminino & 1694 & 68,3 & 66,4 a 70,1 \\
\hline Masculino & 788 & 31,7 & 29,9 a 33,6 \\
\hline Idade & 2482 & & \\
\hline 20-39 anos & 89 & 3,6 & 2,9 a 4,4 \\
\hline 40-59 anos & 831 & 33,5 & 31,6 a 35,4 \\
\hline $60-74$ anos & 1073 & 43,2 & 41,3 a 45,2 \\
\hline $75-84$ anos & 408 & 16,4 & 15,0 a 18,0 \\
\hline 85-101 anos & 81 & 3,3 & 2,6 a 4,0 \\
\hline Escolaridade & 2272 & & \\
\hline Até Fundamental completo/Médio Incompleto & 1771 & 77,9 & 76,2 a 79,6 \\
\hline Médio completo/Superior & 501 & 22,1 & 20,4 a 23,8 \\
\hline ABEP* & 2354 & & \\
\hline $\mathrm{A} / \mathrm{B}$ & 580 & 24,6 & 22,9 a 26,4 \\
\hline $\mathrm{C}$ & 1513 & 64,3 & 62,3 a 66,2 \\
\hline $\mathrm{D} / \mathrm{E}$ & 261 & 11,1 & 9,9 a 12,4 \\
\hline Situação Conjugal & 2482 & & \\
\hline Com companheiro & 1301 & 52,4 & 50,4 a 54,4 \\
\hline Sem companheiro & 1181 & 47,6 & 45,6 a 49,6 \\
\hline \multicolumn{4}{|c|}{ Mais alguém mora na sua casa com o mesmo diagnóstico } \\
\hline HAS** & 2306 & & \\
\hline Sim & 1062 & 46,1 & 44,0 a 48,1 \\
\hline Não & 1244 & 53,9 & 51,9 a 56,0 \\
\hline $\mathrm{DM}^{* * *}$ & 823 & & \\
\hline Sim & 195 & 23,7 & 20,8 a 26,8 \\
\hline Não & 628 & 76,3 & 73,3 a 79,2 \\
\hline
\end{tabular}

*ABEP: Associação Brasileira de Empresas de Pesquisa; **HAS: Hipertensão; ***DM: Diabetes. 
Tabela 2. Caracterização dos problemas de saúde dos hipertensos e diabéticos atendidos no SSC/GHC.

\begin{tabular}{|c|c|c|c|}
\hline Características & Frequência (n) & Percentual (\%) & $\begin{array}{l}\text { Intervalo de Confiança } \\
\qquad(95 \%)\end{array}$ \\
\hline Problema de Saúde & 2482 & & \\
\hline HAS* & 1655 & 66,7 & 64,8 a 68,5 \\
\hline $\mathrm{DM}^{* *}$ & 160 & 6,4 & 5,5 a 7,5 \\
\hline $\mathrm{HAS}^{*} \mathrm{DM}^{* * *}$ & 667 & 26,9 & 25,1 a 28,7 \\
\hline \multicolumn{4}{|l|}{ Tempo de diagnóstico } \\
\hline HAS* & 2313 & & \\
\hline Até 1 ano & 150 & 6,5 & 5,5 a 7,6 \\
\hline Até 2 anos & 160 & 6,9 & 5,9 a 8,0 \\
\hline Acima de 2 anos & 2003 & 86,6 & 85,1 a 88,0 \\
\hline $\mathrm{DM}^{* *}$ & 822 & & \\
\hline Até 1 ano & 84 & 10,2 & 8,2 a 12,5 \\
\hline Até 2 anos & 67 & 8,2 & 6,4 a 10,2 \\
\hline Acima de 2 anos & 671 & 81,6 & 78,8 a 84,2 \\
\hline Complicações (infarto do coração, derrame cerebral e/ou amputação de membro) & 2421 & & \\
\hline Sim & 398 & 16,4 & 14,6 a 17,5 \\
\hline Não & 2023 & 83,6 & 79,9 a 83,0 \\
\hline Percepção de saúde & 2474 & & \\
\hline Excelente & 36 & 1,5 & 1,2 a 2,0 \\
\hline Muito Boa & 98 & 3,9 & 3,2 a 4,8 \\
\hline Boa & 1014 & 40,9 & 38,9 a 42,8 \\
\hline Regular & 1050 & 42,3 & 40,4 a 44,9 \\
\hline Ruim & 276 & 11,1 & 9,9 a 12,4 \\
\hline
\end{tabular}

*HAS: Hipertensão; ** DM: Diabetes; ***HAS*DM: Hipertensão e diabetes.

Tabela 3. Fatores de risco para Doenças Cardiovasculares associados ao estilo de vida dos hipertensos e diabéticos atendidos no SSC/GHC.

\begin{tabular}{|c|c|c|c|}
\hline Características & Frequência (n) & Percentual (\%) & $\begin{array}{l}\text { Intervalo de Confiança } \\
\qquad(95 \%)\end{array}$ \\
\hline Tabagismo & 2482 & & \\
\hline Não, nunca fumou & 1303 & 52,5 & 50,5 a 54,5 \\
\hline Já fumei, mas parei de fumar & 803 & 32,4 & 30,5 a 34,2 \\
\hline Sim, fumo (mais de 1 cigarro por dia há mais de 1 mês) & 376 & 15,1 & 13,8 a 16,6 \\
\hline Adequação Alimentar & 2263 & & \\
\hline Adequada & 522 & 23,0 & 21,3 a 24,9 \\
\hline Parcialmente Adequada & 1052 & 46,0 & 44,4 a 48,6 \\
\hline Inadequada & 689 & 30,0 & 28,6 a 32,3 \\
\hline Uso problemático do álcool & 2482 & & \\
\hline Sim & 124 & 5,0 & 4,2 a 5,9 \\
\hline Não & 2358 & 95,0 & 94,1 a 95,8 \\
\hline Sedentarismo & 2482 & & \\
\hline Sim & 1291 & 52,0 & 50,0 a 54,0 \\
\hline Não & 1191 & 48,0 & 46,0 a 50,0 \\
\hline Estado Nutricional & 2471 & & \\
\hline Baixo Peso & 31 & 1,3 & 0,9 a 1,8 \\
\hline Eutrofia & 722 & 29,2 & 27,4 a 31,1 \\
\hline Pré-obeso & 965 & 39,1 & 37,1 a 41,0 \\
\hline Obesidade I & 516 & 20,9 & 19,3 a 22,5 \\
\hline Obesidade II & 237 & 9,6 & 8,5 a 10,8 \\
\hline
\end{tabular}

A utilização do serviço de saúde está descrita na Tabela 4, onde se observa que pouco mais da metade dos hipertensos e diabéticos havia consultado com o médico nos últimos seis meses, enquanto menos de um quarto tinha consultado com o enfermeiro e menos de um quinto com o dentista. Entre os diabéticos, 10\% consultou com o nutricionista. O tempo de agendamento foi menor do que 15 dias para a grande maioria. Poucos afirmaram ter recebido visita domiciliar de um profissional de saúde $(11 \%)$ e $5 \%$ participavam de grupos de HAS ou DM. Mais de três quartos dos usuários classificou o serviço de saúde como bom. 
Tabela 4. Utilização e satisfação com o serviço por hipertensos e diabéticos no SSC/GHC em 2011.

\begin{tabular}{|c|c|c|c|}
\hline Utilização do SSC & Frequência (n) & Percentual (\%) & $\begin{array}{l}\text { Intervalo de Confiança } \\
\qquad(95 \%)\end{array}$ \\
\hline \multicolumn{4}{|l|}{ Consultas Médicas últimos 6 meses } \\
\hline Por HAS* & 2321 & & \\
\hline Nenhuma & 858 & 37,0 & 35,0 a 39,0 \\
\hline 1 consulta & 518 & 22,3 & 20,6 a 24,1 \\
\hline 2 consultas & 348 & 15,0 & 13,6 a 16,5 \\
\hline 3-6 consultas & 521 & 22,4 & 20,8 a 24,2 \\
\hline$\geq 7$ consultas & 77 & 3,3 & 2,6 a 4,1 \\
\hline Por $\mathrm{DM}^{* *}$ & 826 & & \\
\hline Nenhuma & 267 & 32,3 & 29,1 a 35,6 \\
\hline 1 consulta & 182 & 22,0 & 19,2 a 25,0 \\
\hline 2 consultas & 150 & 18,2 & 15,6 a 21,0 \\
\hline 3-6 consultas & 203 & 24,6 & 21,7 a 27,7 \\
\hline$\geq 7$ consultas & 24 & 2,9 & 1,9 a 4,3 \\
\hline \multicolumn{4}{|l|}{ Tempo de espera: agendamento até consulta } \\
\hline HAS* $^{*}$ & 1241 & & \\
\hline$\leq 15$ dias & 1010 & 81,4 & 79,1 a 83,5 \\
\hline$>15$ dias & 231 & 18,6 & 16,5 a 20,9 \\
\hline $\mathrm{DM}^{* *}$ & 492 & & \\
\hline$\leq 15$ dias & 395 & 79,5 & 75,6 a 83,0 \\
\hline$>15$ dias & 101 & 20,5 & 17,0 a 24 \\
\hline \multicolumn{4}{|l|}{ Acompanhamento regular com o enfermeiro } \\
\hline HAS* & 2174 & & \\
\hline Sim & 488 & 22,4 & 20,7 a 24,3 \\
\hline Não & 1686 & 77,6 & 75,7 a 79,3 \\
\hline $\mathrm{DM}^{* *}$ & 776 & & \\
\hline Sim & 186 & 24,0 & 21,0 a 27,1 \\
\hline Não & 590 & 76,0 & 72,9 a 79,0 \\
\hline Consulta com o dentista & 2481 & & \\
\hline Sim & 356 & 14,3 & 13,0 a 15,8 \\
\hline Não & 2125 & 85,7 & 84,2 a 87,0 \\
\hline \multicolumn{4}{|l|}{ Acompanhamento regular com o Nutricionista } \\
\hline $\mathrm{DM}^{* *}$ & 783 & & \\
\hline Sim & 75 & 9,6 & 7,6 a 11,9 \\
\hline Não & 708 & 90,4 & 88,1 a 92,4 \\
\hline Visita Domiciliar no último mês & 2482 & & \\
\hline Sim & 271 & 10,9 & 9,7 a 12,2 \\
\hline Não & 2211 & 89,1 & 87,8 a 90,3 \\
\hline Participação em grupos & 2455 & & \\
\hline Sim & 122 & 4,9 & 4,1 a 5,9 \\
\hline Não & 2333 & 95,0 & 94,1 a 95,9 \\
\hline Satisfação com o atendimento & 2479 & & \\
\hline Ruim & 294 & 11,9 & 10,6 a 13,2 \\
\hline Regular & 297 & 12,0 & 10,7 a 13,3 \\
\hline Bom & 1888 & 76,2 & 74,4 a 76,8 \\
\hline
\end{tabular}

*HAS: Hipertensão; ** DM: Diabetes.

\section{DISCUSSÃO}

A maioria dos usuários do SSC/GHC com HAS ou DM são mulheres, com proporções bem próximas às relatadas em outros estudos realizados com o programa de Saúde da Família em diferentes localidades, como em um município do Estado de São Paulo, com 68,1\%, no município de Blumenau com $67,5 \%$, ambos realizados em $2006^{19,20}$ e no município de Porto Alegre, com 63,3\%, realizado em $2009^{21}$.
Uma das razões para a superioridade feminina nos serviços de saúde parece ser uma maior percepção do seu estado de saúde-doença e maior tendência ao autocuidado e a busca de auxílio médico para si e para seus familiares ${ }^{22}$.

A amostra apresentou maior proporção de idosos (60\% dos usuários), semelhante a um trabalho realizado na Cidade de Paranoá-DF no ano de 2011, que encontrou $65 \%{ }^{23}$, o que vai ao encontro da situação das DCNT no país. Existe influência da idade na prevalência de DM, pois foi observado 
incremento de $2,7 \%$ na faixa etária de 30 a 59 anos para $17,4 \%$ na de $60-69$ anos, ou seja, um aumento de 6,4 vezes ${ }^{6}$. No Brasil, dados da Vigilância de Fatores de Risco e Proteção para Doenças Crônicas por Inquérito Telefônico (Vigitel), de 2011, apontam também que o DM aumenta de acordo com a idade da população: 21,6\% dos brasileiros com mais de 65 anos referiram a doença, um índice bem maior do que entre as pessoas na faixa etária entre 18 e 24 anos, em que apenas $0,6 \%$ relataram ter diabetes ${ }^{24}$. Em relação à HAS, existe relação direta e linear da pressão arterial com a idade, sendo a prevalência de HAS superior a $60 \%$ acima de 65 anos $^{8}$.

Entre os entrevistados, observou-se baixa escolaridade, $78 \%$ tem o ensino fundamental completo, estes dados coincidem com outras pesquisas realizadas no município de Blumenau no ano de 2008 e na Cidade de Paranoá-DF em 2011, onde foram encontrados $74,8 \%{ }^{19}$ e $81 \%{ }^{23}$ de usuários com escolaridade até ensino fundamental, respectivamente. A prevalência de HAS e DM é mais comum em pessoas com baixa escolaridade, mostrando que o nosso resultado vai ao encontro de outros estudos realizados ${ }^{24}$. Os números indicam que $7,5 \%$ das pessoas que têm até oito anos de estudo possuem diabetes, contra $3,7 \%$ das pessoas com mais de 12 anos de estudo, uma diferença de mais de $50 \%{ }^{25}$. No Brasil, a HAS foi mais prevalente entre indivíduos com menor escolaridade $^{8}$. Entre as mulheres, destaca-se a associação inversa entre nível de escolaridade e diagnóstico: enquanto $34,4 \%$ das mulheres com até 8 anos de escolaridade referiam HAS, a mesma condição foi observada em apenas 14,2\% das mulheres com 12 ou mais anos de escolaridade. Para os homens, o diagnóstico da doença foi menos frequente nos que estudaram de 9 a 11 anos $^{26}$. A baixa escolaridade é um fator preocupante em se tratando de pacientes crônicos que necessitam manter um controle em relação à sua medicação e alimentação. Com base nesses dados, é importante que a equipe de saúde busque ações educativas adequadas para o ensino e aprendizagem dos indivíduos a fim de facilitar a compreensão dos cuidados necessários para o controle da doença e prevenção de complicações ${ }^{22}$.

$\mathrm{Na}$ presente pesquisa, $52 \%$ dos usuários viviam com companheiro e $64 \%$ eram da classe $C$, dados semelhantes foram encontrados em um estudo realizado em Pelotas, RS, no ano de 2009 , onde 53,3\% viviam com companheiro e 60,6\% dos participantes eram pertencentes à classe econômica $\mathrm{C}^{27}$. Ao avaliar a prevalência de doenças crônicas autorreferidas com as características sociodemográficas, a prevalência de problemas crônicos foi inversamente proporcional à renda familiar. Os indivíduos que tinham até um salário mínimo de renda familiar mensal apresentaram prevalência de problemas crônicos $41 \%$ maior do que aqueles cuja renda era maior do que 20 salários mínimos ${ }^{28}$.
Quanto à caracterização do problema de saúde, este trabalho mostrou que $67 \%$ eram só hipertensos, $27 \%$ apresentavam os dois problemas associados e $6 \%$ eram só diabéticos, sendo o tempo de diagnóstico superior a dois anos para a maior parte dos hipertensos e diabéticos. Estudo realizado no México, onde foi avaliada a participação das enfermeiras no cuidado de doentes crônicos na atenção primária à saúde, encontrou resultados distintos, sua população correspondeu a 44,9\% de hipertensos, $28,1 \%$ com ambas as doenças e $27 \%$ somente eram diabéticos ${ }^{29}$. Já, ao avaliar o perfil de hipertensos e diabéticos de três unidades básicas de saúde do município de Pelotas, sul do Brasil, observou-se maior ocorrência de hipertensão com $63 \%$ dos indivíduos, seguidos pelos hipertensos e diabéticos com $31,3 \%$, enquanto que apenas $2,5 \%$ foram diagnosticados com diabetes ${ }^{22}$. Ao analisar a condição de saúde dos indivíduos no interior de São Paulo atendidos em uma equipe de saúde da família, encontrou-se $60 \%$ de hipertensos, $28 \%$ de hipertensos e diabéticos e apenas $12 \%$ eram diabéticos. Ao avaliar o tempo diagnóstico, 36\% dos hipertensos referiram período superior a 10 anos e 33,5\% dos diabéticos referiram tempo de 1 a 2 anos $^{30}$.

Baixo índice de complicações foi encontrado na presente pesquisa (infarto agudo do miocárdio, acidente vascular cerebral e amputação em diabéticos) ao levar em consideração a alta prevalência de fatores de risco. Em um estudo realizado no município de São Carlos, foram avaliados 2.662 cadastros do HiperDia, mostrando uma prevalência de $80 \%$ de hipertensos e diabéticos e $20 \%$ de diabéticos. Ao analisar a presença de complicações, foram encontradas maiores índices do que a presente pesquisa, como: doença renal, pé diabético e amputação por diabetes, em 9,6, 5,1 e 2,4\%, respectivamente. Dentre as doenças cardiovasculares, $8 \%$ foram acidente vascular cerebral (AVC), 11\% infarto agudo do miocárdio (IAM) e outras coronariopatias foram observados em $13 \%{ }^{31}$. Assim como, ao analisar 1.193 fichas de pessoas cadastradas no HiperDia em um município do sul do Brasil foi encontrado como complicações: cardiopatias presentes em 24,2\%, IAM e AVC em 11,6\%, doença renal em $6,1 \%$, pé diabético em $2,5 \%$ e amputação por diabetes em 1,7\% $\%^{22}$.

A autopercepção de saúde baseia-se em critérios subjetivos e objetivos, refletindo uma percepção individual, que inclui aspectos biológicos, psicológicos e sociais. É influenciada pelas condições socioeconômicas, sexo, idade e presença de doenças crônicas ${ }^{32}$. Além disso, deve-se considerar o impacto que essas doenças geram no bem-estar físico, mental e social dos indivíduos ${ }^{33}$. É essencial entender como a pessoa percebe sua saúde, pois o seu comportamento é condicionado pela percepção e pela importância dada a 
esta $^{31}$. Se for positiva, possibilita maior envolvimento dos indivíduos no tratamento e controle da sua doença ${ }^{34}$.

Na população estudada, grande parte considerou sua saúde regular (42\%) ou boa (41\%). Observou-se que mais da metade dos entrevistados não tem uma boa percepção de saúde, ou seja, considera regular ou ruim, o que pode ter relação com a alta prevalência de fatores de risco. Esses achados diferem dos encontrados em um estudo que avaliou a qualidade de vida de hipertensos e diabéticos, onde 53,3\% classificaram sua saúde como regular e $26,7 \%$ boa $^{34}$.

Os fatores relacionados aos hábitos de vida têm influência direta no estabelecimento e controle da HAS e DM, e têm sido considerados fatores de risco para mortalidade prematura ${ }^{22}$. Esta pesquisa apresentou como resultados altos índices de fatores de risco. Em um estudo realizado com hipertensos e diabéticos no município de Santa Rosa, no Rio Grande do Sul, cadastrados no posto de saúde, ao analisar os fatores de risco, foram encontrados como resultados: $16,7 \%$ eram tabagistas e 39,6\% eram sedentários. Ao avaliar o estado nutricional, este estudo mostrou maior prevalência de obesidade com $49,8 \%$, seguido de sobrepeso com $34,2 \%$ e eutrofia com $16 \%{ }^{35}$. Dados superiores ao presente estudo foram encontrados em relação ao índice de sobrepeso e obesidade, correspondendo a $79,4 \%$ dos registros do HiperDia ${ }^{31}$.

O tabagismo é um fator de risco para seis das oito principais causas de morte no mundo (doença isquêmica do coração, acidente vascular cerebral, infecção respiratória baixa, doença pulmonar obstrutiva crônica, tuberculose, câncer de pulmão, brônquios e traqueia). Os efeitos prejudiciais do tabagismo nas complicações diabéticas, particularmente na nefropatia e na morbidade e mortalidade macrovascular, estão bem estabelecidos. Aumenta também o risco de complicações cardiovasculares secundárias em hipertensos e aumenta a progressão da insuficiência renal ${ }^{36}$. Os sedentários perdem os inúmeros efeitos positivos que a atividade física regular traz no metabolismo de lipídios e da glicose, na pressão arterial, na densidade óssea, na produção de hormônios e antioxidantes, no trânsito intestinal e nas funções psicológicas ${ }^{36}$.

Ao avaliar o consumo alimentar, a presente pesquisa encontrou que $76 \%$ não apresentaram uma alimentação adequada. No estudo de Guandalini, realizado no município de Matão/SP, em 2010, ao verificar o cumprimento das orientações dietéticas (mudança de hábito alimentar, redução de peso, sal e açúcar) em sua amostra de hipertensos e diabéticos, registrados nas unidades de saúde da família, constatou que $62 \%$ não se alimentavam corretamente ${ }^{30}$. Quanto à análise dietética em um estudo que avaliou a assistência prestada a usuários hipertensos e diabéticos em uma equipe de Saúde da Família de São Paulo, foram encontrados baixos índices de adequação alimentar. Apenas $32,8 \%$ dos usuários apresentaram alimentação adequada, $42,3 \%$ parcialmente adequada e $23,8 \%$ inadequada ${ }^{20}$. Em uma pesquisa que avaliou a adequação dos hábitos alimentares, realizada com a mesma base de dados do presente estudo, foi verificado baixo consumo de fibras e alto consumo de açúcar, sal e gordura. E encontrou maior inadequação dos hábitos alimentares nos hipertensos $(32,5 \%)$ e a adequação foi maior em quem apresentava HAS e DM $(26,8 \%)^{37}$.

A alimentação está relacionada diretamente a alguns fatores que interferem na prevenção e/ou controle das doenças crônicas e seus agravos. São eles: excesso de peso, dislipidemia, mau controle glicêmico e padrão alimentar com consumo excessivo de gordura saturada e pouca ingestão de frutas e vegetais. Dessa forma, a orientação nutricional é útil na prevenção para o retardo das complicações associadas às condições crônicas. As modificações na alimentação são reconhecidas como um recurso para o controle glicêmico e redução do risco das doenças cardiovasculares ${ }^{36}$.

De acordo com o protocolo do Serviço de Saúde Comunitária do Grupo Hospitalar Conceição, para os hipertensos e diabéticos, a periodicidade do acompanhamento em consulta médica encontra-se dentro do previsto, visto que a maioria apresentou 1 consulta em 6 meses. Já o número de consultas com os enfermeiros foi abaixo do preconizado, pois mais da metade dos entrevistados não haviam realizado uma consulta com estes profissionais ${ }^{38,39}$. Os enfermeiros têm responsabilidade importante na educação dos pacientes sobre os seus problemas de saúde, autocuidado e prevenção de danos. Dessa forma, é fundamental ampliar o acesso com estes profissionais para a qualidade do cuidado ${ }^{41}$.

Ao avaliar a assistência prestada em um serviço de atenção primária à saúde do município de Santa Rosa, RS, para hipertensos e diabéticos, foi encontrado maior número de consultas, $87,5 \%$ com uma consulta ou mais com o médico de família e comunidade (MFC) para hipertensos e de três consultas ou mais para $60 \%$ dos diabéticos. Estes dados correspondem a uma cobertura de $59,6 \%$ para HAS e $64,8 \%$ para DM, considerada boa pelos autores do estudo. Para este serviço do interior do Rio Grande do Sul, foi considerado como meta para hipertensos uma consulta médica por ano e para os diabéticos três consultas médicas por ano $^{35}$. Não foram encontrados estudos que avaliassem o número de consultas com os profissionais da enfermagem.

O tempo de agendamento foi menor do que 15 dias para a maioria dos hipertensos e diabéticos. Em um estudo realizado em São Paulo, o tempo de agendamento para 
consultas médicas em unidades de saúde foi inferior a 15 dias para $61 \%$ dos hipertensos e diabéticos ${ }^{20}$. A demora no tempo de agendamento das consultas constitui um motivo para a procura de atendimento em serviços de urgência e emergência, interferindo no seguimento da assistência à saúde e no estabelecimento de vínculo entre o usuário e o profissional. É importante para os pacientes com doenças crônicas, o acompanhamento adequado na unidade de saúde de referência, para melhor controle da HAS e DM e prevenção de complicações decorrentes da sua condiçãa ${ }^{42}$.

Foi encontrado um baixo número de consultas com os dentistas, de acordo com o Protocolo de cuidado de hipertensos e diabéticos na atenção primária do Grupo Hospitalar Conceição. Este, recomenda que os pacientes de baixo risco devem realizar pelo menos uma consulta de avaliação e continuar o acompanhamento conforme a avaliação inicial. Se moderado e alto risco, a consulta deve ser anual. Todos os profissionais deveriam orientar uma consulta com o cirurgião-dentista, conforme fluxo de atendimento de cada unidade de saúde ${ }^{39}$. Esse cuidado é importante, pois a presença de infecções agudas e condições inflamatórias na cavidade bucal podem aumentar a taxa de glicose. E a doença periodontal (gengivite e periodontite) é a principal manifestação odontológica do portador de $\mathrm{DM}^{43}$.

O nutricionista não está presente nas equipes de saúde da família, pois faz parte dos Núcleos de Apoio a Saúde da Família (NASF). A proposta é que 1 NASF sirva de apoio para 8 a 20 equipes de saúde da família, surtindo efeitos positivos no processo de educação permanente, porém sendo insuficiente para a atenção multiprofissional ${ }^{40}$. Dessa forma, o baixo número de consultas com estes profissionais encontrados neste estudo, com $10 \%$ de consultas para os diabéticos, se justifica, como regra geral, pois não há consultas individuais com os nutricionistas e sim atividades com o objetivo de promoção de alimentação saudável e controle das doenças relacionadas à alimentação ${ }^{38}$. No estudo de Guandalini ${ }^{30}$, 17\% dos hipertensos e diabéticos passaram pelo serviço de nutrição.

Segundo Vilaça, a atenção às condições crônicas deve envolver uma equipe multiprofissional, a fim de prestar atendimentos programados individuais ou em grupos. Devem incluir a atenção às agudizações das condições crônicas, ações preventivas, educacionais e de autocuidado apoiado. As intervenções individuais e grupais podem promover o empoderamento das pessoas e capacitá-las para o autocuidado, sendo estratégias efetivas no manejo das condições crônicas ${ }^{40}$.

A participação em grupos de educação em saúde nas unidades foi muito menor da encontrada no estudo de Souto et al., correspondendo a $27,2 \%{ }^{32}$. A baixa frequência às atividades de grupo deve servir de alerta sobre a adequação do trabalho nessa modalidade assistencial (estrutura disponível, acesso e tipos de atividades realizadas), pois estudos nacionais demonstraram que esta modalidade, quando bem planejada e com sustentação ao longo do tempo, pode contribuir para melhoria de parâmetros clínicos, bem como de educação em saúde e contribuir para constituição de redes de apoio social ${ }^{19}$. Há vários benefícios para a utilização de grupos nas unidades: redução dos atendimentos individuais, participação ativa das pessoas no processo de mudança, maior envolvimento dos profissionais com as pessoas usuárias e estímulo à autonomia das pessoas na produção da saúde ${ }^{44}$.

Poucos (11\%) afirmaram ter recebido visita domiciliar de um profissional de saúde no último mês. Esse dado mostra a dificuldade já relatada em outros estudos sobre a organização dos profissionais para realização de visitas domiciliares. A ESF prevê a atenção domiciliar como forma de assistência àqueles que precisam de cuidados contínuos, além de servir como um instrumento para o diagnóstico local e programação das ações a partir da realidade. Favorece também a formação de vínculo com a população, a integralidade, humanização e responsabilização dos profissionais ${ }^{45}$. Segundo a Política Nacional de Atenção Básica (PNAB), os agentes comunitários de saúde devem realizar visitas domiciliares às famílias de sua responsabilidade uma vez por mês, avaliando a necessidade de aumentar a frequência ${ }^{46}$.

A satisfação dos usuários é um componente importante na qualidade do cuidado, pois permite a avaliação e possível aprimoramento dos serviços oferecidos ${ }^{47}$. Neste estudo foram encontrados altos índices de satisfação com o serviço de saúde. Ao avaliar a assistência prestada ao paciente pela estratégia saúde da família, encontrou que $80 \%$ dos diabéticos classificaram o serviço prestado como muito bom ou bom ${ }^{23}$. A satisfação com a assistência prestada a hipertensos e diabéticos foi de $68,8 \%$ em uma equipe de saúde do município de São Paulo ${ }^{20}$.

Este estudo apresentou limitações relacionadas às perguntas autorreferidas dependentes da memória dos entrevistados, o que pode ter acarretado em vieses de informação. Considerando que parte da amostra selecionada não pertencia, no momento da entrevista, à população-alvo do estudo, as perdas e recusas foram estimadas em cerca de $25 \%$, o que pode eventualmente enviesar os resultados encontrados.

Observou-se neste estudo que grande parte da amostra de hipertensos e/ou diabéticos eram mulheres, idosas, com baixa escolaridade e pertencentes à classe econômica C. A presença de hábitos de vida, que são considerados fatores de risco, como o fumo, alimentação inadequada, sedentarismo, 
sobrepeso e obesidade, mostrou-se bastante prevalente. O número de consultas com os médicos foi de acordo com o preconizado pelo protocolo do Serviço de Atenção Primária do Grupo Hospitalar Conceição. Porém, observou-se baixa utilização da equipe multiprofissional (consulta com enfermeiro e dentista, visitas domiciliares e participação em grupos). O uso do serviço precisa melhorar, visto que para o atendimento de doenças crônicas, é necessário trabalhar com grupos de educação em saúde e com uma equipe multiprofissional, o que não foi encontrado nesta pesquisa. Encontrou-se também que mais da metade não tem uma boa percepção de saúde, apesar dos baixos índices de complicações da sua condição. Grande parte dos usuários está satisfeito com o atendimento prestado pelas equipes de Saúde da Família.

Análises mais aprofundadas desses dados devem buscar fatores associados aos problemas aqui descritos. Além disso, sugere-se que novas pesquisas sejam realizadas a fim de entender quais foram os aspectos considerados pelos indivíduos na satisfação com o serviço de saúde e também quais são os fatores responsáveis pela percepção de saúde das pessoas.

\section{REFERÊNCIAS}

1. World Health Organization. World Health Statistics 2012 [Internet]. Geneva: WHO; 2012 [cited 2016]. p. Available from: http://apps. who.int/iris/bitstream/10665/44844/1/9789241564441_eng.pdf

2. Freitas LRS, Garcia LP. Evolução da prevalência do diabetes e deste associado à hipertensão arterial no Brasil: análise da Pesquisa Nacional por Amostra de Domicílios, 1998, 2003 e 2008. Epidemiol Serv Saúde. 2012;21(1):7-19. http://dx.doi.org/10.5123/S167949742012000100002

3. Rocha-Brischiliari SC, Agnolo CMD, Gravena AAF, Lopes TCR, Carvalho MD de B, Pelloso SM. Doenças Crônicas não Transmissíveis e associação com fatores de risco. Rev Bras Cardiol. 2014;27(1): 531-8.

4. Oliveira-Campos M, Rodrigues-Neto JF, Silveira MF, Neves DMR, Vilhena JM, Oliveira JF, Magalhães JC, Drumond D. Impacto dos fatores de risco para doenças crônicas não transmissíveis na qualidade de vida. Cien Saude Colet. 2013;18(3):873-82. http:// dx.doi.org/10.1590/S1413-81232013000800033

5. Schmidt MI, Duncan BB, Hoffmann JF, Moura L de, Malta DC, Carvalho RMSV. Prevalência de diabetes e hipertensão no Brasil baseada em inquérito de morbidade auto-referida, Brasil, 2006. Rev Saude Publica. 2009;43(Supl 2):74-82. http://dx.doi.org/10.1590/ S0034-89102009000900010

6. Sociedade Brasileira de Diabetes. Diretrizes da Sociedade Brasileira de Diabetes 2013-2014 [Internet]. São Paulo: AC Farmacêutica; 2014 [cited 2016]. Available from: http://www.nutritotal.com.br/ diretrizes/files/342--diretrizessbd.pdf

7. Moraes AS, Freitas ICM, Gimeno SGA, Mondini L. Prevalência de diabetes mellitus e identificação de fatores associados em adultos residentes em área urbana de Ribeirão Preto, São Paulo, Brasil,
2006: Projeto OBEDIARP. Cad Saúde Pública. 2010;26(5):929-41. http://dx.doi.org/10.1590/S0102-311X2010000500015

8. Sociedade Brasileira de Cardiologia. Departamento de Hipertensão Arterial. VI Diretrizes Brasileiras de Hipertensão. Rev Bras Hipertens. 2010;17(1):69.

9. Gerais FDM, Ho rizonte-mg B, Assis TD, Barreto SM. Hipertensão arterial no Brasil : estimativa de prevalência a partir de estudos de base populacional. Epidemiol Serv Saúde. 2006;15(1):35-45.

10. Moura EC, Silva SA da, Malta DC, Neto OLM. Fatores de risco e proteção para doenças crônicas: vigilância por meio de inquérito telefônico, VIGITEL, Brasil, 2007. Cad Saude Publica. 2011;27(3):48696. http://dx.doi.org/10.1590/S0102-311X2011000300009

11. Capilheira MF, Santos IS, Azevedo Jr. MR, Reichert FF. Risk factors for chronic non-communicable diseases and the CARMEN Initiative : a population-based study in the South of Brazil. Cad Saúde Pública. 2008;24(12):2767-74. http://dx.doi.org/10.1590/S0102$311 \times 2008001200005$

12. Starfield B. Atenção primária equilíbrio entre necessidades de saúde, serviços e tecnologia. Brasília: Ministério da Saúde; 2002.

13. Ministério da Saúde (BR). Secretaria de Vigilância em Saúde. Departamento de Análise de Situação de Saúde. Coordenação Geral de Doenças e Agravos Não Transmissíveis. Plano de Ações Estratégicas para o Enfrentamento das Doenças Crônicas Não Transmissíveis (DCNT) no Brasil 2011-2022 [Internet]. Brasília, DF: Ministério da Saúde; 2011 [cited 2016]. Available from: http://bvsms.saude.gov.br/bvs/publicacoes/plano_acoes_enfrent dcnt_2011.pdf

14. Ramos DD, Lima MADS. Acesso e acolhimento aos usuários em uma unidade de saúde de Porto Alegre, Rio Grande do Sul, Brasil. Cad Saúde Pública. 2003;19(1):27-34. http://dx.doi.org/10.1590/ S0102-311X2003000100004

15. Ministério da Saúde (BR). Guia alimentar: como ter uma alimentação saudável [Internet]. Brasília, DF: Ministério da Saúde; 2006 [cited 2016]. Available from: http://bvsms.saude.gov.br/bvs/publicacoes/ guia_alimentar_populacao_brasileira_2008

16. Shayesta D, Jacek K. The CAGE questionnaire for alcohol misuse: A review of reliability and validity studies. Clin Invest Med. 2007;30(1):33-41.

17. Benedetti TRB, Antunes PC, Rodriguez-Anez CR, Mazo GZ, Petrosk EL. Reprodutibilidade e validade do Questionário Internacional de Atividade Física (IPAQ) em homens idosos. Rev Bras Med Esporte. 2007;13:1. http://dx.doi.org/10.1590/S1517-86922007000100004

18. World Health Organization. Obesity: preventing and managing the global epidemic. Report of a World Health Organization Consultation. Geneva: WHO; 2000. WHO Obesity Technical Report Series, no 284.

19. Barros $A C M$, Rocha MB, Helena ETIS. Adesão ao tratamento e satisfação com o serviço entre pessoas com diabetes mellitus atendidas no PSF em Blumenau, Santa Catarina. ACM Arq Catarin Med. 2006;37(1):54-62

20. Paiva DCP de, Bersusa AAS, Escuder MML. Avaliação da assistência ao paciente com diabetes e/ou hipertensão pelo Programa Saúde da Família do Município de Francisco Morato, São Paulo, Brasil. Cad Saude Publica. 2006;22(2):377-85. http://dx.doi.org/10.1590/ S0102-311X2006000200015 
21. Zils AA, Castro RCL de, Oliveira MMC de, Harzheim E, Duncan BB. Satisfação dos usuários da rede de Atenção Primária de Porto Alegre. Rev Bras Med Fam Com. 2009;4(16):270-6. http://dx.doi. org/10.5712/rbmfc4(16)233

22. Lima LM de, Schwartz E, Muniz RM, Zillmer JGV, Ludtke I. Perfil dos usuários do hiperdia de três unidades báscias de saude do sul do Brasil. Rev Gaúcha Enferm. 2011;32(2):323-9. http://dx.doi. org/10.1590/S1983-14472011000200016

23. Pereira JN, Lucio LM, Silva TC. Avaliação da assistência prestada ao paciente com diabetes mellitus tipo 2, pela Estratégia Saude da Família no 6, da cidade do Paranoá-DF, Brasil. Rev APS. 2013;16(4):350-6.

24. Ministério da Saúde (BR). Secretaria de Atenção à Saúde. Departamento de Atenção Básica. Cadernos de Atenção Básica Estratégias para o cuidado da pessoa com doença crônica Diabetes Mellitus [Internet]. Brasília, DF: Ministério da Saúde; 2013 [cited 2016]. Available from: http://189.28.128.100/dab/docs/portaldab/ publicacoes/caderno_36.pdf

25. Ministério da Saúde (BR). Secretaria de Vigilância em Saúde. Departamento de Análise de Situação de Saúde. Saúde Brasil 2011 Uma análise da situação de saúde e a vigilância da saúde da mulher [Internet]. Brasília, DF: Ministério da Saúde; 2012 [cited 2016]. Available from: http://bvsms.saude.gov.br/bvs/publicacoes/ saude_brasil_2011.pdf

26. Ministério da Saúde (BR). Secretaria de Atenção à Saúde. Departamento de Atenção Básica. Cadernos de Atenção Básica Estrtatégias para o cuidado da pessoa com doença crônica Hipertensão Arterial Sistêmica [Internet]. Brasília, DF: Ministério da Saúde; 2013 [cited 2016]. Available from: http://189.28.128.100/ dab/docs/portaldab/publicacoes/caderno_37.pdt

27. Azevedo ALS, Silva RA, Tomasi E, Quevedo L Á. Doenças crônicas e qualidade de vida na atenção primária à saúde. Cad Saúde Pública. 2013;29(9):1774-82. http://dx.doi.org/10.1590/S0102$311 \times 2013001300017$

28. Almeida MF, Barata RB, Montero CV, Silva ZP. Prevalência de doenças crônicas auto-referidas e utilização de serviços de saúde, PNAD / 1998, Brasil. Ciênc Saúde Colet. 2002;7(4):743-56. http:// dx.doi.org/10.1590/s1413-81232002000400011

29. Pérez-cuevas R, Morales HR, Doubova SV, Arias MZ, Rodríguez GD, Valdovinos AP, Hernández OM. Atención integral de pacientes diabéticos e hipertensos con participación de enfermeras en medicina familiar. Rev Panam Salud Públ. 2009;26(6):511-7. http:// dx.doi.org/10.1590/S1020-49892009001200006

30. Guandalini VR. Estratégia Saúde da Família: avaliação dos cuidados em saúde e nutrição aos adultos diabéticos e hipertensos, Matão/SP [tese]. São Paulo: UNESP; 2013.

31. Jardim ADI, Leal AMO. Qualidade da informação sobre diabéticos e hipertensos registrada no Sistema HIPERDIA em São Carlos - SP, 2002-2005. Physis Rev Saúde Colet. 2009;2(19):2002-5. http:// dx.doi.org/10.1590/s0103-73312009000200009

32. Agostinho MR, Oliveira MC, Pinto MEB, Balardin GU, Harzheim E. Autopercepção da saúde entre usuários da Atenção Primária em Porto Alegre, RS. Rev Bras Med Fam Comunidade. 2010;5(51):9-15. http://dx.doi.org/10.5712/rbmfc5(17)175

33. Ministério da Saúde (BR). Secretaria de Vigilância em Saúde. Vigitel Brasil 2013. Vigilância de Fatores de Risco e Proteção para Doenças Crônicas por Inquérito Telefônico [Internet]. Brasília, DF: Ministério da Saúde; 2014. [cited 2016]. Available from: http://bvsms.saude. gov.br/bvs/publicacoes/vigitel_brasil_2013.pdf
34. Miranzi S de SC, Ferreira FS, Iwamoto HH, Pereira G de A, Miranzi MAS. Qualidade de vida de indivíduos com diabetes mellitus e hipertensão acompanhados por uma equipe de saúde da família. Texto Contexto Enferm. 2008;17(4):672-9. http://dx.doi. org/10.1590/s0104-07072008000400007

35. Souto CO, Silbermann CD, Aita GL, Benvegnu LA. Avaliação da assistência a diabéticos e ou hipertensos em uma unidade de Atenção Primária à Saúde. Rev Bras Med Fam Comunidade. 2009;4(16):260-9. http://dx.doi.org/10.5712/rbmfc4(16)232

36. Ministério da Saúde (BR). Secretaria de Atenção à Saúde. Departamento de Atenção Básica. Cadernos de Atenção Básica Estratégias para o cuidado da pessoa com doença crônica [Internet]. Brasília, DF: Ministério da Saúde; 2014 [cited 2016]. Available from: http://189.28.128.100/dab/docs/portaldab/publicacoes/ caderno_35.pdf

37. Lima LA de, Nedel FB, Olinto MTA, Baldisserotto J. Food habits of hypertensive and diabetics cared for in a Primary Health Care Service in the South of Brazil. Rev Nutr. 2015;28(2):197-206. http:// dx.doi.org/10.1590/1415-52732015000200008

38. Ferreira SRS, Bianchini IM, Flores R. A Organização do cuidado às pessoas com hipertensão arterial sistêmica em Serviços de Atenção Primária à Saúde. Porto Alegre: Grupo Hospitalar Conceição; 2011.

39. Ferreira SRS, Bianchini IM, Flores R. A organização do cuidado às Pessoas com diabetes mellitus tipo 2, em Serviços de Atenção Primária à Saúde. Porto Alegre: Grupo Hospitalar Conceição; 2011.

40. Mendes EV. As redes de atenção à saúde [Internet]. 2 ${ }^{a}$ ed. Brasília, DF: Organização Pan-Americana da Saúde; 2011 [cited 2016]. Available from: http://apsredes.org/site2012/wp-content/ uploads/2012/03/Redes-de-Atencao-mendes2.pdf

41. Franzen E, Almeida MA, Aliti G, Bercini RR, Menegon DB, Rabelo ER. Adultos e idosos com doenças crônicas: implicações para o cuidado de enfermagem. Rev HCPA. 2007;27(2):28-31.

42. Souza MF, Figueiredo LA, Pinto IC. Análise da utilização do serviço de pronto-socorro na percepção do usuário. Cienc Cuid Saude. 2010;9(1):13-20. http://dx.doi.org/10.4025/cienccuidsaude. v9i1.5242

43. Terra BG, Goulart RR, Bavaresco CS. O Cuidado odontológico do paciente portador de diabetes mellitus tipo 1 e 2 na atenção primária à saúde. Rev APS. 2011;14(2):149-61 .

44. Mendes EV. O cuidado das condições crônicas na atenção primária à saúde: o imperativo da consolidação da estratégia da saúde da família [Internet]. Brasília, DF: Organização Pan-Americana da Saúde; 2012 [cited 2016]. Available from: http://bvsms.saude. gov.br/bvs/publicacoes/cuidado_condicoes_atencao_primaria_ saude.pdf

45. Cunha MS, Sá MC. A visita domiciliar na Estratégia de Saúde da Família: os desafios de se mover no território. Interface (Botucatu). 2013;17(44):61-73. http://dx.doi.org/10.1590/S141432832013000100006

46. Ministério da Saúde (BR). Secretaria de Atenção à Saúde. Departamento de Atenção Básica. Política Nacional de Atenção Básica [Internet]. Brasília, DF: Ministério da Saúde; 2012. Available from: http://189.28.128.100/dab/docs/publicacoes/geral/ pnab.pdf

47. Cotta RMM, Marques ES, Maia TM, Azeredo CM, Schott M, Franceschini SC, Priore SE. A satisfação dos usuários do Programa de Saúde da Família : avaliando o cuidado em saúde. Sci Med (Porto Alegre). 2005;15(4):227-34 